\title{
Parallel Computation of RCS of Electrically Large Platform with Coatings Modeled with NURBS Surfaces
}

\author{
Ying Yan, ${ }^{1}$ Wei-Xin Zhao, ${ }^{2}$ Xun-Wang Zhao, ${ }^{1}$ Yu Zhang, ${ }^{1}$ and Chang-Hong Liang1 \\ ${ }^{1}$ Science and Technology on Antenna and Microwave Laboratory, Xidian University, Shaanxi, Xi'an 710071, China \\ ${ }^{2}$ Department of Electrical Engineering and Computer Science, Syracuse University, Syracuse, NY 13244-1240, USA
}

Correspondence should be addressed to Ying Yan, yymqn1008@163.com

Received 23 December 2011; Revised 9 April 2012; Accepted 11 April 2012

Academic Editor: Tat Yeo

Copyright ( 2012 Ying Yan et al. This is an open access article distributed under the Creative Commons Attribution License, which permits unrestricted use, distribution, and reproduction in any medium, provided the original work is properly cited.

\begin{abstract}
The significance of Radar Cross Section (RCS) in the military applications makes its prediction an important problem. This paper uses large-scale parallel Physical Optics (PO) to realize the fast computation of RCS to electrically large targets, which are modeled by Non-Uniform Rational B-Spline (NURBS) surfaces and coated with dielectric materials. Some numerical examples are presented to validate this paper's method. In addition, 1024 CPUs are used in Shanghai Supercomputer Center (SSC) to perform the simulation of a model with the maximum electrical size $1966.7 \lambda$ for the first time in China. From which, it can be found that this paper's method can greatly speed the calculation and is capable of solving the real-life problem of RCS prediction.
\end{abstract}

\section{Introduction}

Since the invention of radar, many applications have been found for its use in military, which include the detection and the tracking of ships, aircrafts, and ground vehicles. Modern radar systems make it virtually impossible for vehicles to approach targets without being detected at great distance. For this single reason, the military has put great emphasis on the reduction of RCS of military vehicles. The word "stealth" has been used to describe military vehicles specially designed with a low RCS.

PO technique is a powerful tool to analyze the target with electrically large dimensions, especially to predict its RCS. However, many researches of PO technique focus on the problem of target with PEC surface, not only the triangular surface modeling technique [1-6] but also the NURBS modeling technique [7-10], but the study of PO theory to calculate the body with dielectric coatings is not comprehensive. As we know, the platform usually has dielectric coatings on its surface with different aims. Specially, in the military applications, coating on the surface has become a more and more significant method to reduce the vehicle's RCS. Therefore, this paper studies the PO theory to calculate RCS of target with dielectric coatings. Moreover, there also have been some research papers that describe the PO technique to compute RCS of body with coatings [11-14], but most of them are serial significant code. When the model simulated has very large electrical dimensions, the simulation time will be very long, and the computational efficiency will be pretty low as well. To make the PO technique for model with coatings more practical, this paper also proposes a large-scale parallel scheme, which is based on MPI (Message Passing Interface) [15] library. And also a test in SSC [16] using 1024 CPUs is performed.

In addition, as a popular modeling technique, NURBS [7-10] is used to achieve the object of accurate modeling.

In conclusion, this paper realizes the rapid prediction of RCS to electrically large platform coated with dielectric materials by using more than 1000 CPUs. In Section 2, the basic theory and computational platforms are presented; Section 3 presents some numerical examples to validate the accuracy and effectiveness of this paper's approach; finally, Section 4 makes the conclusion.

\section{Basic Theory}

2.1. Initializing of the NURBS Model. As we know, NURBS is a very powerful tool for geometric design due to several reasons including fast and numerically stable algorithms and 


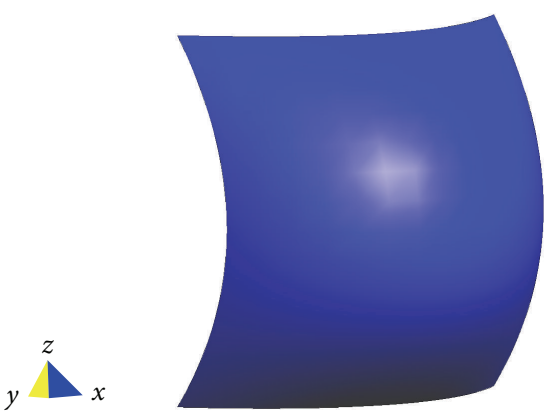

(a) Model of a Rational Bezier surface

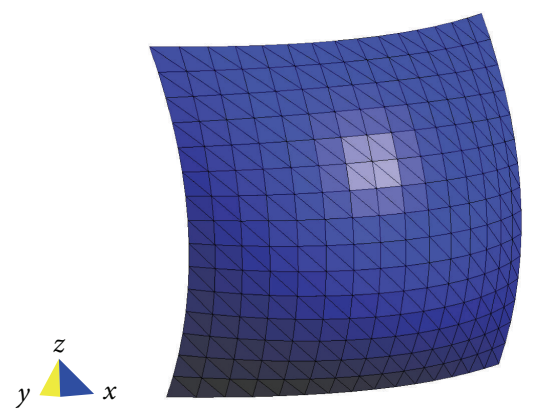

(b) Both directions of $U$ and $V$ have 15 segments

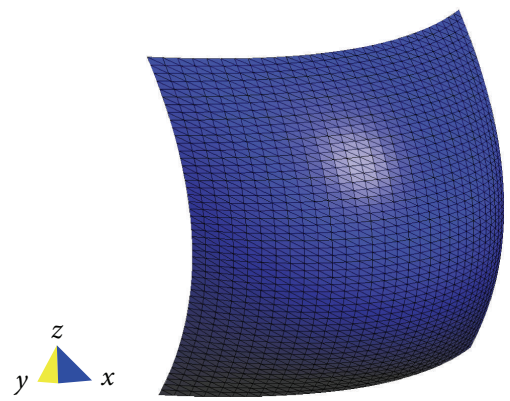

(c) Direction $U$ has 30 segments and $V$ has 50 segments

FIGURE 1: Illustrating of Rational Bezier surfaces with different mesh sizes.

unified mathematical basis for representing complex objects [17]. Using it to model target geometry is good for target with curved and smooth surfaces so as to guarantee the accuracy of the model and make it more realistic.

However, although target modeling is easy with NURBS, applying some numerical tools directly on NURBS is not so straightforward. Since the surface point function and derivative computation is simpler for Rational Bezier surfaces, NURBS surfaces are usually converted to Rational Bezier surfaces by Cox-de Boor Algorithm [18], which can be directly realized by the commercial modeling software, such as Rhinoceros. Therefore, in this paper, Rational Bezier surfaces are used for surface modeling.

Before the simulation, we should firstly generate a NURBS model using the commercial software, such as Rhinoceros. In this case, NURBS model is flexible for arbitrary target. "obj" is a file format to store the NURBS model and the file of this format is simple to handle in the computation. Therefore, this paper uses this format to store the data of input model.

The initialing of the NURBS model can be summarized into two steps: (1) the NURBS surfaces composing the model should be converted to Rational Bezier surfaces, and this step can be directly realized by Rhinoceros; (2) the converted model is imported, and the code in this paper can automatically mesh each Bezier surface to a lot of small planar patches, which means that it is not necessary to use any commercial software to do the operation of mesh. In addition, the mesh size of two directions $U$ and $V$, of the Bezier surface can be adjusted to acquire the best effect, which is shown in Figure 1.

As illustrated in Figure 1, it can be found that the mesh size of each direction will affect the accuracy in modeling so that the computational precision will be influenced as well. Therefore, the choice of the mesh sizes in each direction is important.

2.2. RCS Calculation of Target with Dielectric Coatings. Consider the situation depicted in Figure 2. An arbitrary scattered body is placed at the origin, with the observation point being at coordinates $(x, y, z)$. For RCS calculation, the observation point is considered to be in the far field of the target. Therefore, the vectors $\vec{R}$ and $\vec{r}$ are approximately parallel.

The formula to calculate the scattered electric field is [19]

$$
\begin{gathered}
\vec{E}(\vec{r})=\frac{1}{j w \varepsilon \cdot 4 \pi} \iint_{s^{\prime}}\left[\frac{3-k^{2} R^{2}+j 3 k R}{R^{5}} e^{-j k R} \vec{R} \times\left(\vec{R} \times \overrightarrow{J_{s}}\left(\overrightarrow{r^{\prime}}\right)\right)\right. \\
\left.+2 \overrightarrow{J_{s}}\left(\vec{r}^{\prime}\right) \frac{1+j k R}{R^{3}} e^{-j k R}\right] d S^{\prime},
\end{gathered}
$$

where $R=\left|\vec{r}-\vec{r}^{\prime}\right|$. Using the approximate formulas of far field can obtain the expression of scattered integral equation [19]

$$
\begin{gathered}
|k R| \gg 1, \quad R=|\vec{r}-\vec{r}| \approx r-\hat{r} \cdot \vec{r}, \quad|\vec{r}| \gg|\vec{r}| \\
\vec{E}_{s}(r, \theta, \phi)=E_{\theta} \hat{\theta}+E_{\phi} \hat{\phi}=\frac{-j k Z_{0}}{4 \pi} \cdot \frac{e^{-j k R}}{R} \iint_{A} \overrightarrow{J_{s}}(\vec{r}) e^{j k g} d S, \\
\hat{r} \cdot \vec{r}=g .
\end{gathered}
$$

Generally, the magnetic field intensity of the incident wave can be obtained from the electric field intensity, then $\overrightarrow{J_{s}}=2 \hat{n} \times \vec{H}_{i}$ is used to obtain the induced surface current, which can also be wrote in the form of $\vec{J}_{s}=\left(J_{x} \hat{x}+J_{y} \hat{y}+\right.$ $\left.J_{z} \widehat{z}\right) e^{j k h}$, where $h=\hat{r}_{i} \cdot \vec{r}=x u_{i}+y v_{i}+z w_{i}$.

Usually, after the model has been meshed into a lot of small patches, the global coordinate system is not consistent with the patch's local coordinate system. But when the global system is transformed to the local $\left(x^{\prime \prime}, y^{\prime \prime}, z^{\prime \prime}\right)$, each patch will be on its local $x^{\prime \prime} o y^{\prime \prime}$ plane and with $\widehat{z}^{\prime \prime}$ being the unit 


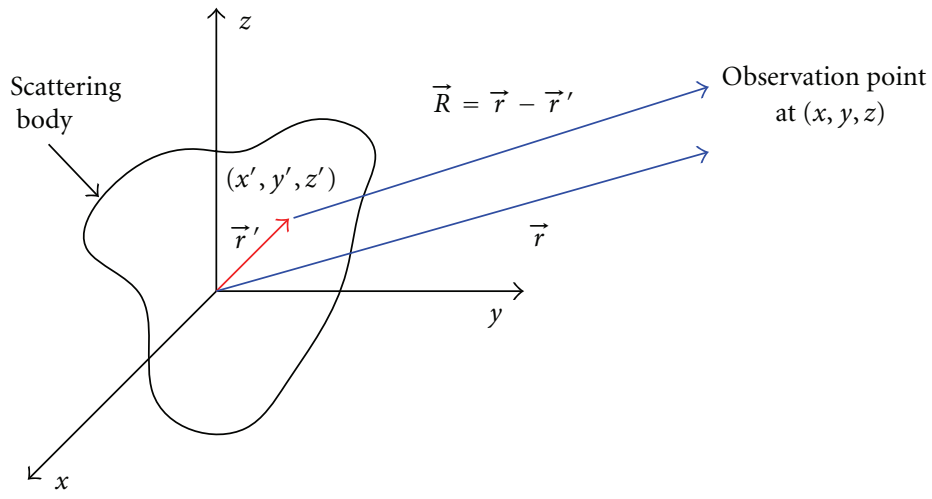

FIGURE 2: Far field scattering from an arbitrary body.

normal vector, and this is convenient for the computation. So we have performed this coordinate transformation and then obtained the induced current $\vec{J}_{s}$ in the local coordinate system:

$$
\begin{gathered}
\overrightarrow{J_{s}}=\left(J_{x}^{\prime \prime} \hat{x}+J_{y}^{\prime \prime} \hat{y}\right) e^{j k h}, \\
h=\hat{r}_{i} \cdot \vec{r}=x u_{i}+y v_{i}+z w_{i} .
\end{gathered}
$$

In (4), the components of surface-induced current $\overrightarrow{J_{x}^{\prime \prime}}, \overrightarrow{J_{y}^{\prime \prime}}$ are $[14,20,21]$

$$
\begin{aligned}
& \overrightarrow{J_{x}^{\prime \prime}}=-\frac{E_{i \theta}^{\prime \prime} \cos \phi^{\prime \prime}}{Z_{0}} \Gamma_{/ /}-\frac{E_{i \phi}^{\prime \prime} \cos \theta^{\prime \prime} \sin \phi^{\prime \prime}}{Z_{0}} \Gamma_{\perp}, \\
& \overrightarrow{J_{y}^{\prime \prime}}=-\frac{E_{i \theta}^{\prime \prime} \sin \phi^{\prime \prime}}{Z_{0}} \Gamma_{/ /}-\frac{E_{i \phi} \cos \theta^{\prime \prime} \cos \phi^{\prime \prime}}{Z_{0}} \Gamma_{\perp} \text {, } \\
& \Gamma_{/ /}=\frac{-Z_{0} \cos \theta^{\prime \prime}}{2 R_{s}+Z_{0} \cos \theta^{\prime \prime}}, \\
& \Gamma_{\perp}=\frac{-Z_{0}}{2 R_{s} \cos \theta^{\prime \prime}+Z_{0}},
\end{aligned}
$$

where $R_{s}$ is the surface impedance. When $R_{s}=0$, the surface is a perfect electric conductor (PEC).

It is necessary to indicate that the reflection coefficients $\Gamma_{\|}, \Gamma_{\perp}$ in (5) can only be applied to the model with impedance surface. In the situation of coated model, $\Gamma$ is obtained by using the theory of wave matrix [14]

$$
\begin{aligned}
{\left[\begin{array}{l}
c_{1} \\
b_{1}
\end{array}\right] } & =\prod_{n=1}^{N} \frac{1}{\tau_{n}}\left[\begin{array}{cc}
e^{j \phi_{n}} & \Gamma_{n} e^{-j \phi_{n}} \\
\Gamma_{n} e^{j \phi_{n}} & e^{-j \phi_{n}}
\end{array}\right]\left[\begin{array}{l}
c_{N+1} \\
b_{N+1}
\end{array}\right] \\
& =\left[\begin{array}{ll}
A_{11} & A_{12} \\
A_{21} & A_{22}
\end{array}\right]\left[\begin{array}{l}
c_{N+1} \\
b_{N+1}
\end{array}\right] .
\end{aligned}
$$

In (6), $b_{1}$ and $c_{1}$ are the reflected wave and the incident wave in the first layer of coatings; $b_{N}, c_{N}$ are the reflected wave and the incident wave in the last layer. $\phi_{n}=\beta_{n} t_{n}=\left(2 \pi / \lambda_{n}\right) t_{n}$, with $\lambda_{n}$ being the wavelength in layer $n$ and $t_{n}$ being the thickness of layer $n$. Finally, the overall reflection coefficient is $\Gamma=b_{1} / c_{1}=A_{21} / A_{11}$.
Substituting $\Gamma$ into (5) can obtain the induced surface current in local coordinate system. Obviously, (6) has to be used twice to compute TE and TM situations, respectively.

When the surface current is obtained, the total scattered field can be calculated by substituting $J_{x}^{\prime \prime}, J_{y}^{\prime \prime}$ into (3). And also, it is necessary to mention that the amplitude of the current is approximate constant over the surface. For the reason that this paper's method automatically mesh the NURBS surface into some small patches, and when the mesh size is small enough, each patch will be planar:

$$
\vec{E}^{S}(\vec{r})=\frac{-j k Z_{0}}{4 \pi} \cdot \frac{e^{-j k R}}{R}\left(J_{x}^{\prime \prime} \hat{x}+J_{y}^{\prime \prime} \hat{y}\right) \iint_{s^{\prime}} e^{j k(g+h)} d S^{\prime} .
$$

Finally, Ludwig integral $[22,23]$ formula is used to calculate $I_{c}=\iint_{s^{\prime}} e^{j k(g+h)} d S^{\prime}$. Substituting the integral result into (7) can get the total scattered field in local coordinate system and then transform it back to global coordinate system and RCS is obtained by the formula $\sigma=$ $\lim _{R \rightarrow \infty} 4 \pi R^{2}\left|\overrightarrow{E^{S}}\right|^{2} /\left|\vec{E}^{I}\right|^{2}$.

2.3. Large-Scale Parallel Scheme. As we know, the model has to be meshed into a lot of elements before the computation. When the model is of electrically large dimensions, the number of meshed elements will be large. In this case, the computational quantity will be tremendous when Bistatic RCS with solid angles (both the azimuth angle $\phi$ and the polarizing angle $\theta$ are changing simultaneously) is computed. If the serial code is still used, it will take a long time to perform the simulation, which is not practical in the engineering. Therefore, the large-scale parallel technique is employed, the scheme of which is described as follows.

At a certain frequency, the parallel scheme is divided into two steps: (1) since the direction of incident wave is fixed, the sheltering procedure should be performed once, the computation of induced surface current as well. With this idea in mind, different patches are assigned to different processes periodically, and then each process conducts the sheltering, respectively. This is beneficial to obtain a good load balance. The scheme of assignment is illustrated in Figure 3, which takes 9 patches and 3 processes as an example. Patches 1, 4, and 7 are assigned to process 0 , patches 


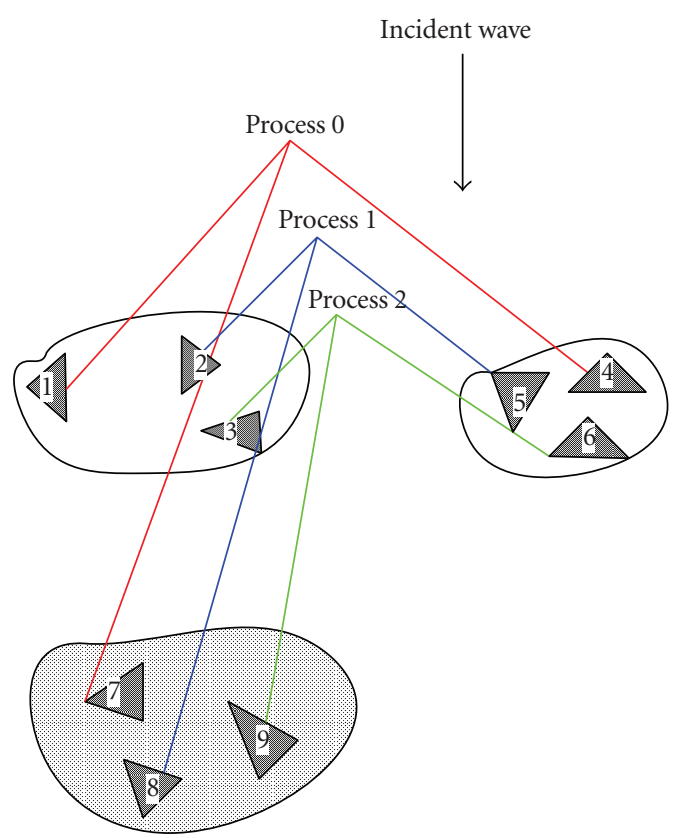

FIGURE 3: Assign patches to different processes periodically.

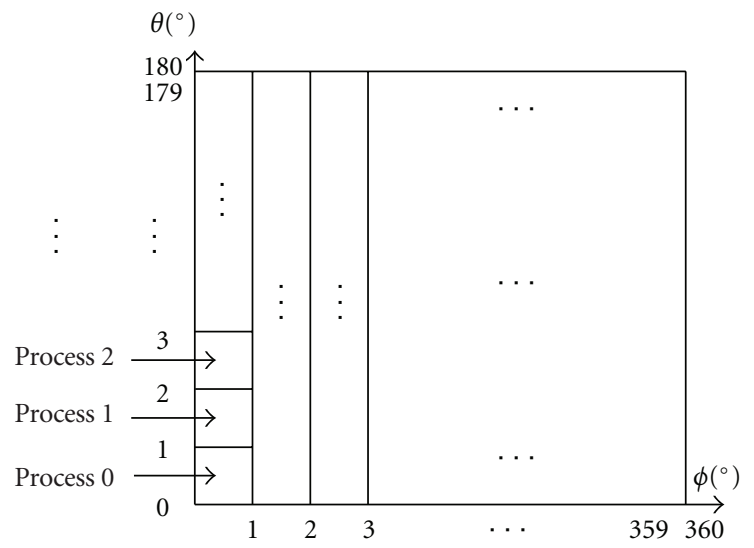

FIGURE 4: Assign scattered angles to different processes periodically.

$2,5,8$ to process 1 , and patches $3,6,9$ to process 2 . When the sheltering procedure is finished, each process will continually calculate the induced surface current, respectively. Finally, all the results of different processes are gathered by the main process 0 ; (2) in scattered direction, all the azimuth angles $\phi$ and the polarizing angles $\theta$ are assigned to all processes periodically, and then they start to calculate the PO integral, respectively, as illustrated in Figure 4. Finally, the results of all the processes are collected by the main process 0 [19].

From what has been presented above, the large-scale parallel scheme is summarized as follows:

(1) MPI initializing;

(2) assign different patches to all the processes periodically, and then each process performs the sheltering procedure and computes the induced surface current, respectively; the main process collects all the

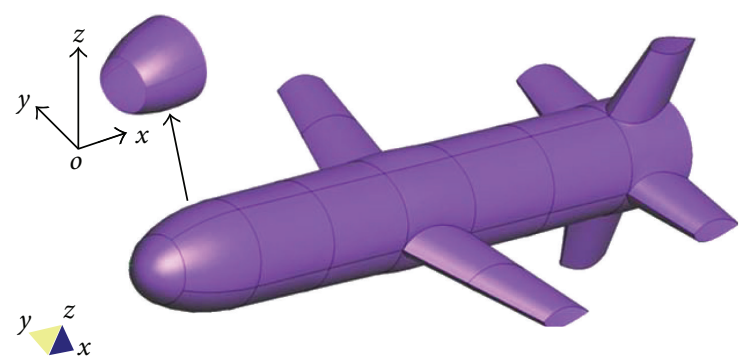

FIGURE 5: RationalBezier surfaces separated from a missile model.

processes' results of sheltering and induced surface current at last;

(3) assign the scattered angles to all the processes periodically, and then each process computes the PO integral and RCS, respectively. Finally, the main process gathers all the processes' results;

(4) MPI terminates.

2.4. Description of the Computational Platforms. To illustrate the versatility of the solver in this paper, two representative computation platforms have been chosen.

(1) Personal Computer: Quad core Intel i5 processor (2.67 GHz) with 4 GB RAM and $500 \mathrm{~GB}$ of hard disk.

(2) Shanghai Supercomputer Center (SSC) [16]: The 37 nodes from Magic-cube Machine with a total of $592 \mathrm{AMD}$ CPU cores $(1.9 \mathrm{GHz}$ per $\mathrm{CPU}$ and 4 cores on each CPU): 16 CPU cores on each node and 4 GB RAM per core, and a total amount of RAM approximately equal to $2.3 \mathrm{~TB}$. No hard disk storage is available for computation. InfiniBand is used for the network's interconnection.

\section{Results and Discussion}

3.1. Results Validation. In this section, the accuracy of this paper's method is validated through three different examples: Section 3.1.1 a missile's head whose surfaces are coated with dielectric materials; Section 3.1.2 an aircraft whose surfaces are coated with dielectric materials; Section 3.1.3 a Rational Bezier surface coated with dielectric materials.

3.1.1. The Model of a Missile's Head. As illustrated in Figure 5, the model simulated is the head of a missile with dielectric materials coated on its surfaces. The model consists of 4 Rational Bezier surfaces and the geometrical dimensions are $8.1 \mathrm{~m} \times 10.1 \mathrm{~m} \times 10.1 \mathrm{~m}$. Its surfaces are coated with a layer of dielectric material, whose parameters are presented in Table 1. The simulation frequency is $300 \mathrm{MHz}$.

In this example, Bistatic RCS is computed, and the results obtained are compared with those from the commercial software FEKO [24] to validate the accuracy of this paper's method. The compared algorithms are chosen to be PO and RWG MoM [25] in FEKO. 
TABLE 1: Information of the material coating.

\begin{tabular}{lccc}
\hline $\begin{array}{l}\text { Relative Electric } \\
\text { Permittivity } \varepsilon_{r}\end{array}$ & $\begin{array}{c}\text { Loss Tangent } \\
\tan \delta\end{array}$ & $\begin{array}{c}\text { Relative magnetic } \\
\text { Permeability } \mu_{r}\end{array}$ & $\begin{array}{c}\text { Thickness } t \\
(\mathrm{~mm})\end{array}$ \\
\hline 16.0 & 0.275 & 1.0 & 5 \\
\hline
\end{tabular}

TABLE 2: Information about the coating.

\begin{tabular}{lcccc}
\hline & $\begin{array}{c}\text { Relative } \\
\text { Electric } \\
\text { Permittivity } \\
\varepsilon_{r}\end{array}$ & $\begin{array}{c}\text { Loss } \\
\text { Tangent } \\
\tan \delta\end{array}$ & $\begin{array}{c}\text { Relative } \\
\text { magnetic } \\
\text { Permeability } \\
\mu_{r}\end{array}$ & $\begin{array}{c}\text { Thickness } \\
t(\mathrm{~mm})\end{array}$ \\
\hline 1st material & 2.5 & 0.00045 & 1.0 & 5 \\
\hline 2nd material & 16 & 0.275 & 1.0 & 6.25 \\
\hline
\end{tabular}

TABLE 3: Comparison of memory required.

\begin{tabular}{lc}
\hline & Memory required (MB) \\
\hline This paper's method & 5.3 \\
PO algorithm in FEKO & 15.3 \\
\hline
\end{tabular}

The incident wave is of VV $(\theta \theta)$ polarization and along the direction of $\theta=0^{\circ}, \phi=0^{\circ}$ and the direction of the scattered wave is $\theta=0^{\circ} \sim 360^{\circ}, \phi=0^{\circ}$.

Figure 6 presents the results' comparison. In Figure 6(a), PO algorithm in FEKO is chosen to be the compared technique to validate this paper's method. It can found from the figure that this paper's results are in good agreement with those of FEKO, except some tiny differences near $\theta=$ $110^{\circ}, 250^{\circ}$; in Figure 6(b), we choose a more exact solutionRWG MoM to compare with this paper's method. From the figure, we can find that the results' comparison among polarizing angles $\theta=20^{\circ} \sim 120^{\circ}$ and $\theta=230^{\circ} \sim 300^{\circ}$ exists some differences; this is reasonable because of the approximation introduced in the PO current. In addition, the results agree well among other angle ranges.

3.1.2. The Model of an Aircraft. In this example, Bistatic RCS of an aircraft coated with dielectric materials is computed. As illustrated in Figure 7, the model's geometrical dimensions are $11.6 \mathrm{~m} \times 7.0 \mathrm{~m} \times 2.92 \mathrm{~m}$, and it is necessary to mention that the model has no structures such as engine and air inlets (i.e., it is not a real aircraft).

(1) Accuracy Validation. In this part, the model is coated with one layer of coating, which is the first kind of material presented in Table 2.

The simulation frequency is $1 \mathrm{GHz}$. The incident wave is of $\mathrm{VV}(\theta \theta)$ polarization and along the direction of the aircraft's nose. The direction of the scattered wave is $\phi=$ $0^{\circ} \sim 360^{\circ}, \theta=90^{\circ}$. This simulation is performed on the first kind of computational platform described in Section 2.4, and the results obtained are compared with those of FEKO. Since this model's electrical dimensions are big and it will take long time to perform the simulation by using RWG MoM, PO algorithm in FEKO is chosen to be the validation method.

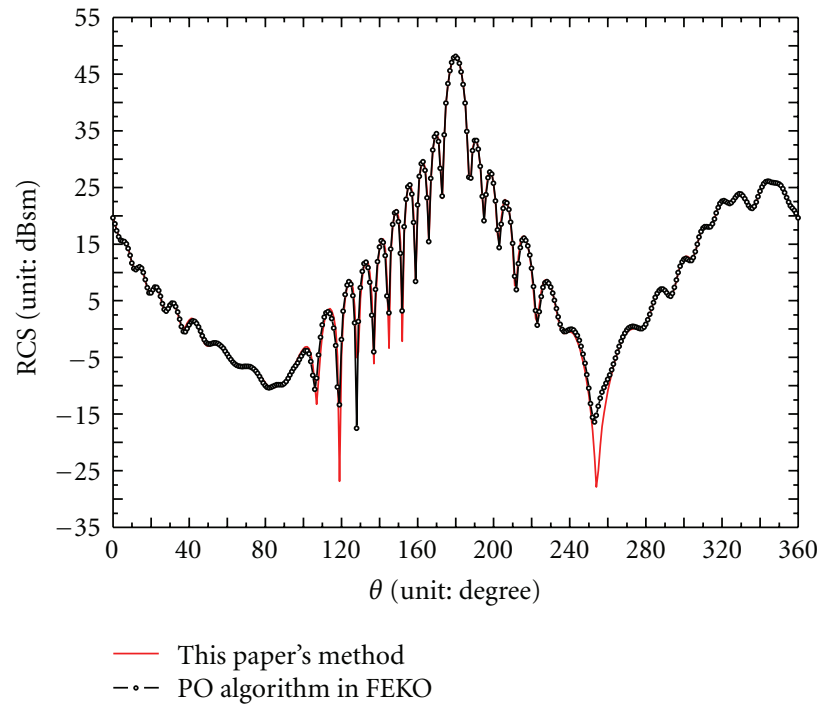

(a) This paper's method versus PO algorithm in FEKO

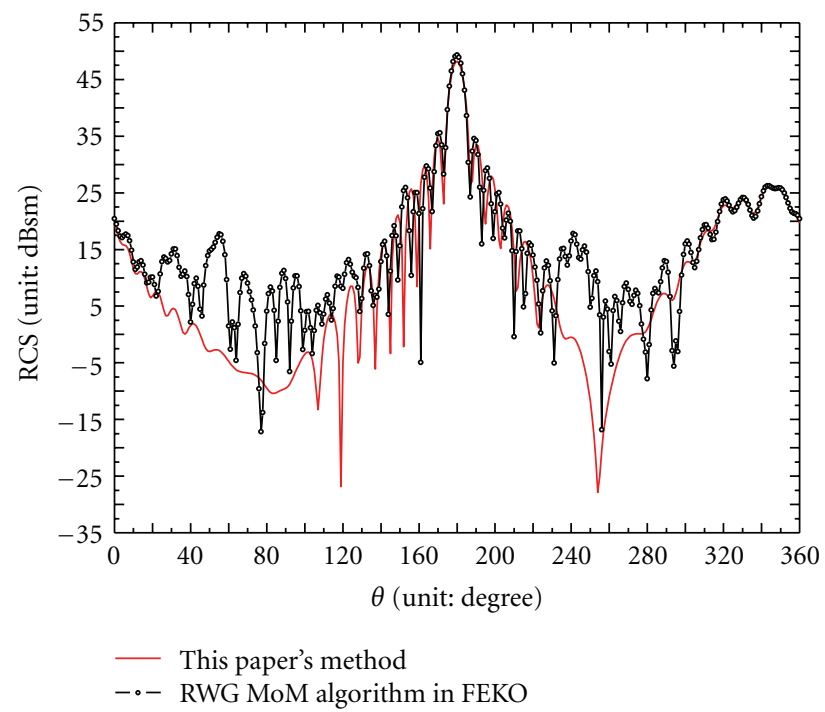

(b) This paper's method versus RWG MoM algorithm in FEKO

Figure 6: Comparison of this paper's results and the results of FEKO.

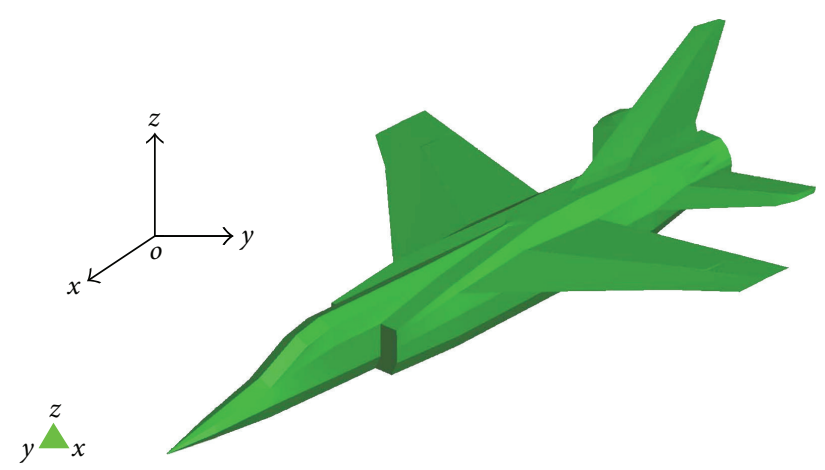

FIGURE 7: Model of an aircraft.

Figure 8 illustrates the comparison of Bistatic RCS on $X O Y$ plane, which indicates that the results of this paper are 


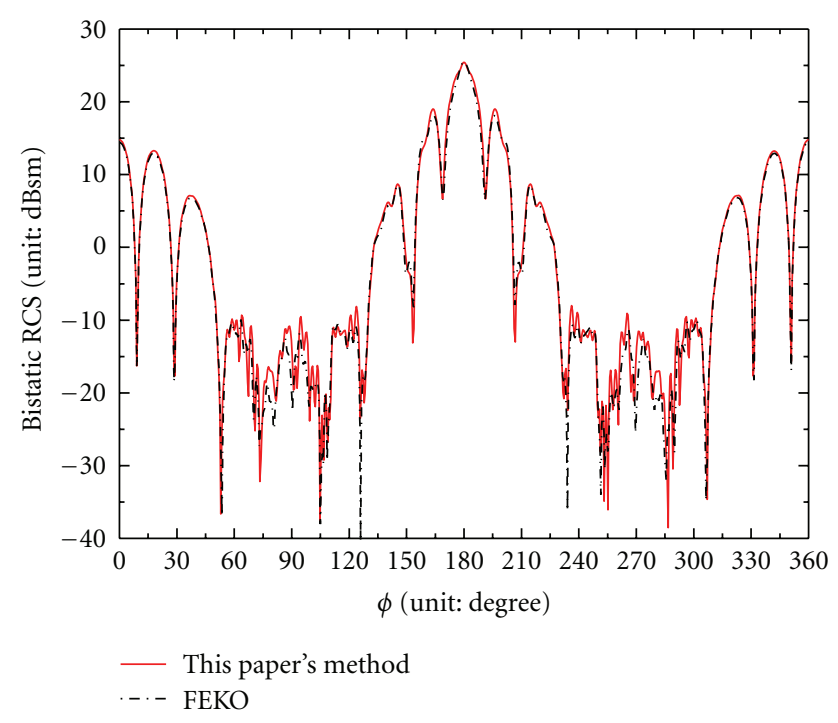

FIgURE 8: This paper's results versus the results of FEKO.

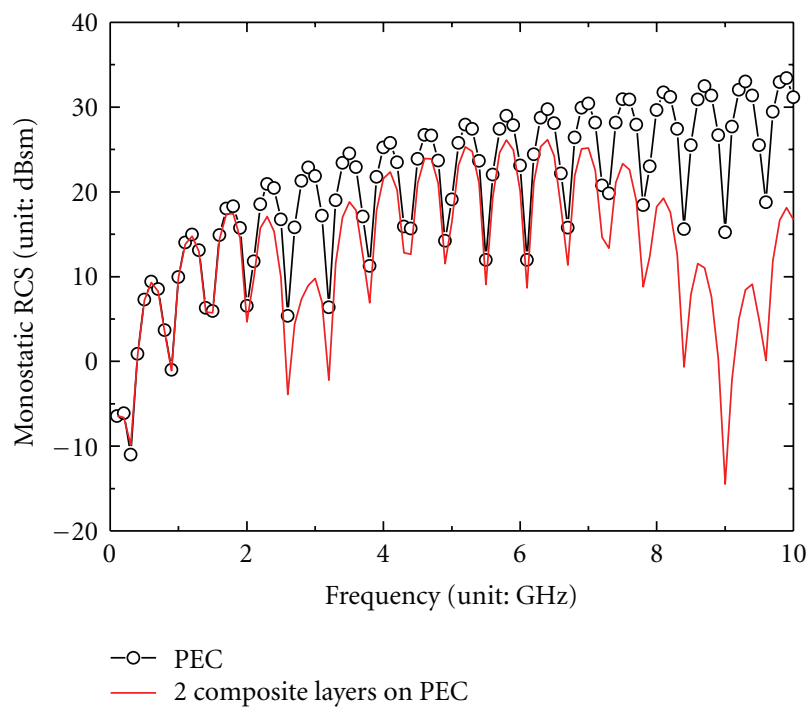

FIgure 9: Aircraft with PEC surface versus Aircraft coated with two layers of materials.

in good agreement with the results of FEKO, which shows that this paper's method is correct.

Moreover, Table 3 compares the memory required in the simulation, which indicates that this paper's method needs less memory than that of FEKO.

(2) RCS Reduction by Using Coatings. In this part, two situations are simulated to compare the variation of RCS: (1) PEC model and (2) model coated with two layers of dielectric materials.

The model simulated is the same as that in part 1 of this example. Two layers of coating, which are the two kinds of materials presented in Table 2, are coated on the aircraft. The frequencies range from $0.1 \mathrm{GHz}$ to $10 \mathrm{GHz}$. The polarization

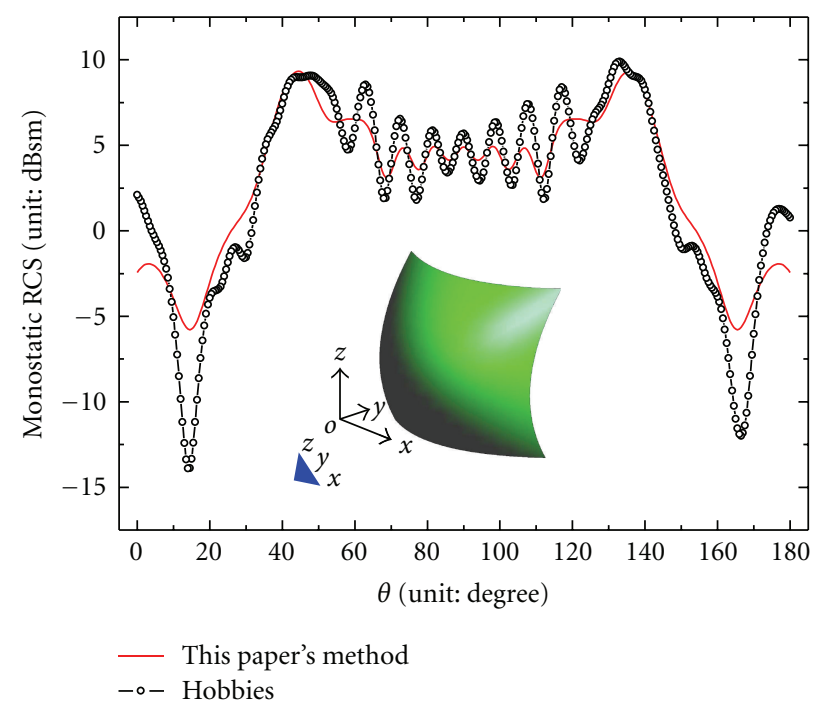

(a) $1 \mathrm{GHz}$

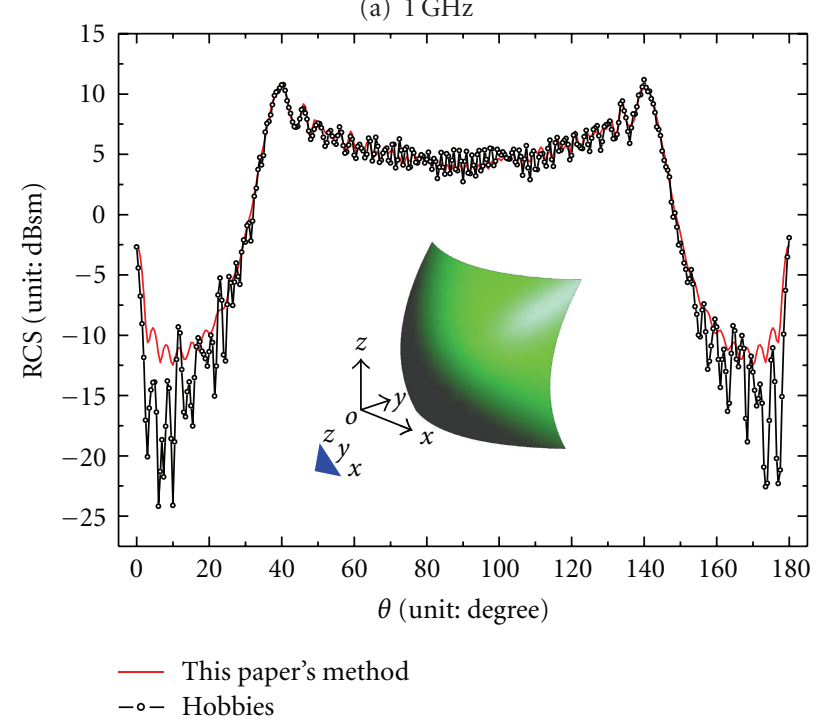

(b) $5 \mathrm{GHz}$

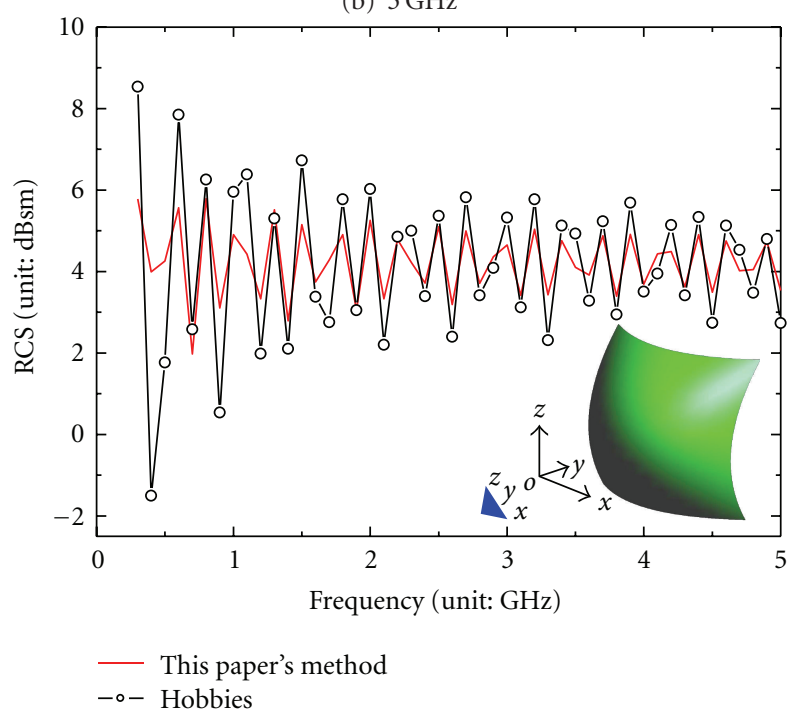

(c) $300 \mathrm{MHz} \sim 5 \mathrm{GHz}$

Figure 10: This paper's method versus Higher-order MoM in HOBBIES. 


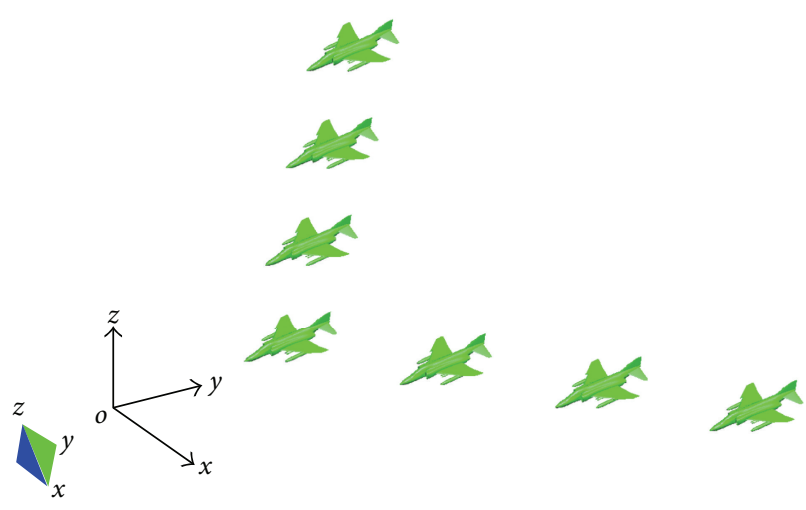

FIGURE 11: A formation of 7 aircrafts.

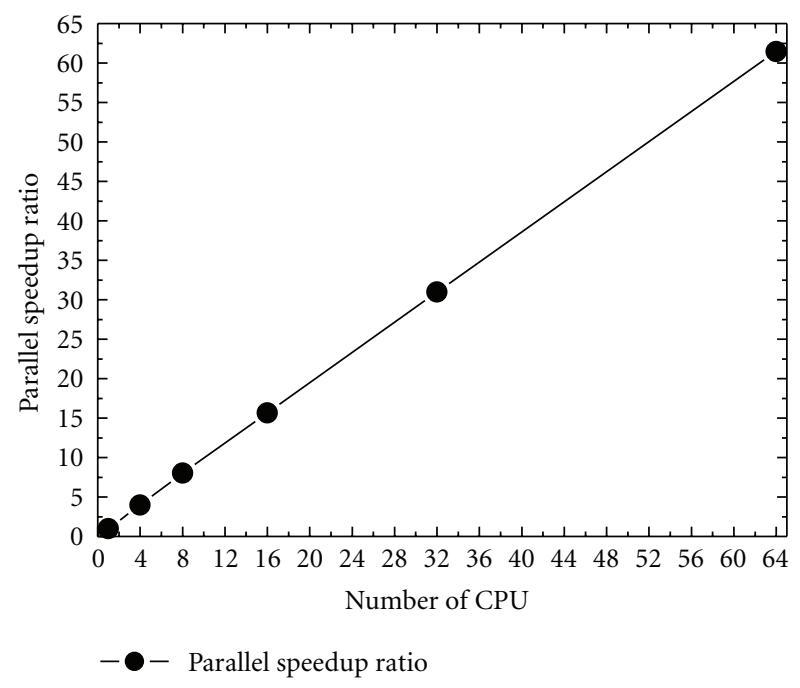

Figure 12: Parallel speed-up ratio.

of the incident wave is $\mathrm{VV}(\theta \theta)$, and the direction of backward scattering is along the nose of the aircraft, namely, $\theta=90^{\circ}, \phi=0^{\circ}$. This simulation is performed on the first kind of computational platform described in Section 2.4.

Figure 9 indicates that two layers of coating will significantly reduce the model's RCS. To be specific, there is a reduction of about $10 \mathrm{~dB}$ at approximate $3 \mathrm{GHz}$, and at the range of $7 \mathrm{~Hz}$ to $10 \mathrm{~Hz}$, RCS is reduced a lot. This example shows the great influence of coating on reducing the target's RCS.

3.1.3. The Model of a Rational Bezier Surface. In this example, a Rational Bezier surface's Monostatic RCS is computed. The results obtained are compared with those from Higher-order MoM [26] in HOBBIES $[27,28]$ to specify in what situation this paper's method is valid.

The model's geometrical dimensions are $2 \mathrm{~m} \times 1.1 \mathrm{~m} \times$ $2 \mathrm{~m}$. It is coated with one layer of material and the parameters of which are presented in Table 1. In Figures 10(a) and $10(\mathrm{~b})$, the simulation frequencies are $1 \mathrm{GHz}$ and $5 \mathrm{GHz}$, respectively. The incident wave is of the $\mathrm{VV}(\theta \theta)$ polarization, and the direction of the backward scattering is $\phi=270 \circ$,

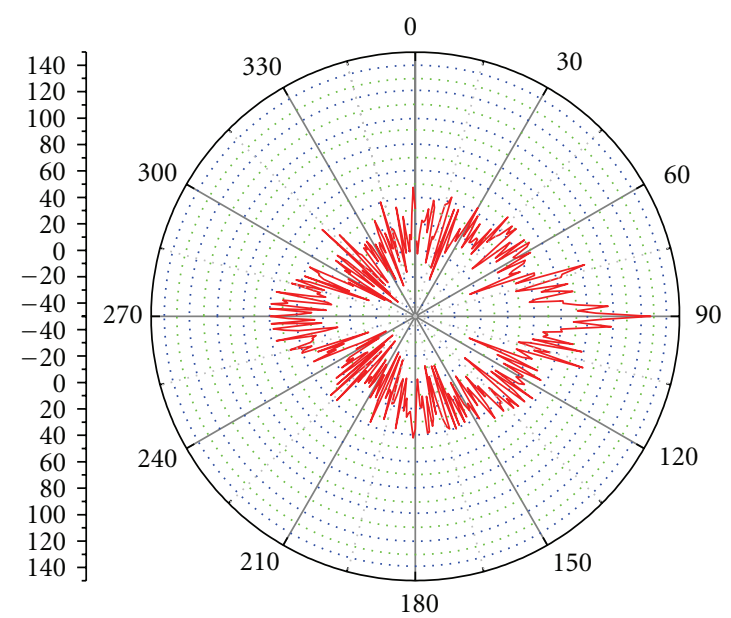

- RCS (unit: dBsm) on XOY plane

(a) XOY plane

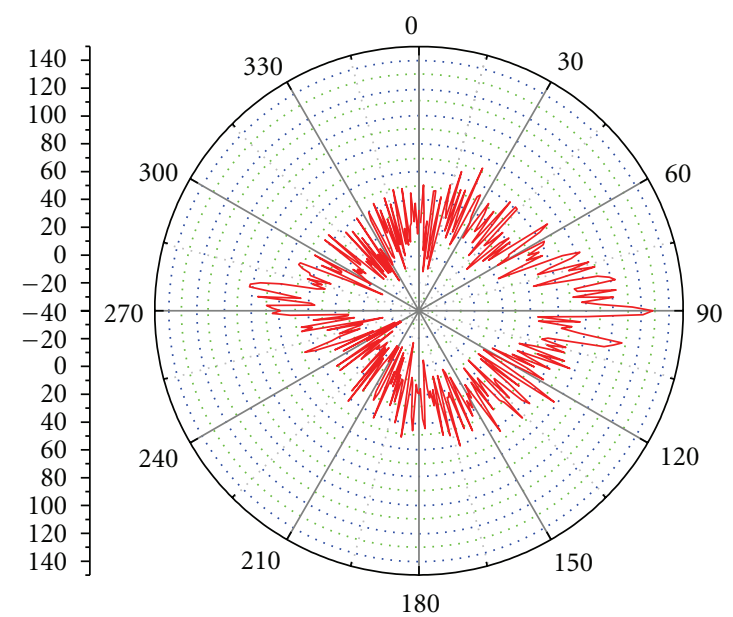

RCS (unit: dBsm) on YOZ plane

(b) YOZ plane

FIgURe 13: Bistatic RCS.

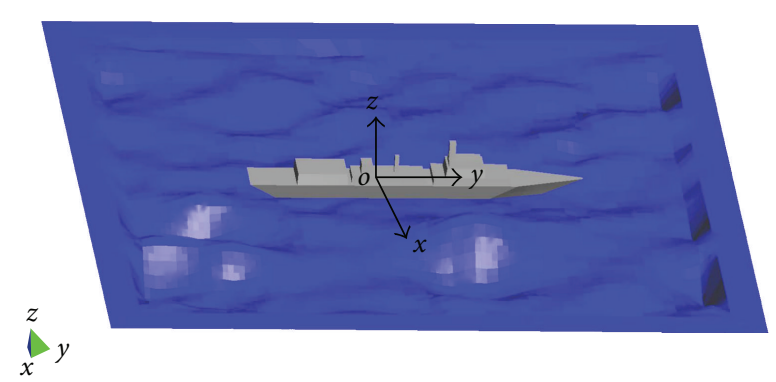

Figure 14: Model of a ship on the sea.

$\theta=0^{\circ} \sim 180^{\circ}$. Figure 10 (c) is to verify the accuracy of this paper's method at various frequencies which range from $300 \mathrm{MHz}$ to $5 \mathrm{GHz}$, and the backward scattering is along $\phi=270^{\circ}, \theta=90^{\circ}$.

From the results illustrated in Figure 10, we can find that both the results of $1 \mathrm{GHz}$ and $5 \mathrm{GHz}$ do not agree well at the 


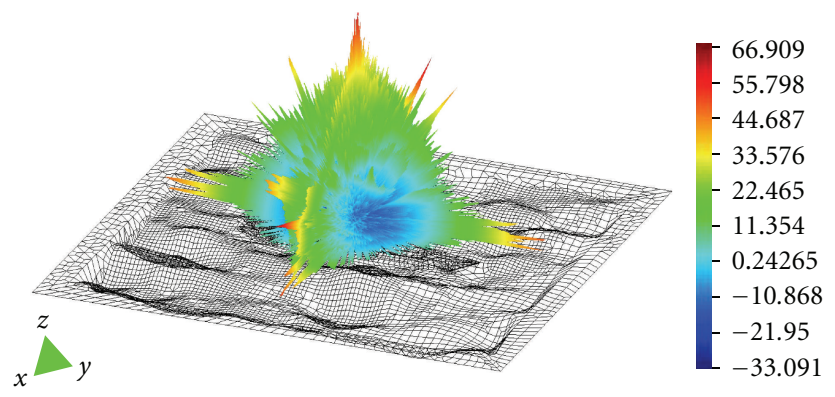

Contour fill of total-RCS $(\mathrm{dB})>$ waveno. $110000 \mathrm{GHz}\left(\sigma / \mathrm{m}^{2}(\mathrm{~dB})\right)$

Figure 15: 3D Monostatic RCS (unit: dBsm).

TABLE 4: Time comparison of using different numbers of CPU.

\begin{tabular}{lcccccc}
\hline Number of CPU & 1 & 4 & 8 & 16 & 32 & 64 \\
\hline $\begin{array}{l}\text { Wall Clock Time } \\
\text { (Second) }\end{array}$ & 164052 & 41054 & 20459 & 10475 & 5293 & 2670 \\
\hline
\end{tabular}

TABLE 5: Dielectric material of the sea.

Relative Electric Loss Tangent Relative magnetic Permeability \begin{tabular}{lcl} 
Permittivity $\varepsilon_{r}$ & $\tan \delta$ & $\mu_{r}$ \\
\hline 81.5 & 0.0 & 1.0
\end{tabular}

angle ranges of $\theta=0^{\circ} \sim 30^{\circ}$ and $\theta=150^{\circ} \sim 180^{\circ}$, which is because there is a structure of edge in the model among these angles, and the diffraction effect that contributes to the scattered field is not considered so that the results near the edge will not be accurate. Moreover, it can also be found that the results of $5 \mathrm{GHz}$ agree better than that of $1 \mathrm{GHz}$ at the angle range of $\theta=30^{\circ} \sim 150^{\circ}$, which indicates that the results will be more accurate when the frequency is higher. Meanwhile, we can also find the better agreement at high frequencies from Figure 10(c).

From what has been described above, this paper's method is accurate when the incident direction is not near the structure of edge of the model and the simulated frequency is high.

3.2. Parallel Speed-Up Ratio. In this example, a formation of 7 aircrafts' Bistatic RCS is calculated by using the largescale parallel technique, which is based on MPI. The model is presented in Figure 11. Each aircraft has the geometrical dimensions of $19.2 \mathrm{~m} \times 12.2 \mathrm{~m} \times 4.3 \mathrm{~m}$ and contains the structure of engine. The model is coated with one layer of material, and the parameters of which are presented in Table 1.

As the simulation frequency is $5.0 \mathrm{GHz}$, the model is automatically meshed into 1094870 elements by this solver. The incident wave of VV $(\theta \theta)$ polarization is along the direction of $\theta=90^{\circ}, \phi=270^{\circ}$, and the scattered direction is $\theta=0^{\circ} \sim 180^{\circ}, \phi=0^{\circ} \sim 360^{\circ}$. The second kind of computational platform described in Section 2.4 is used to perform this test.

Table 4 indicates the time comparison of using different numbers of CPU: it takes 164052 seconds by using $1 \mathrm{CPU}$,

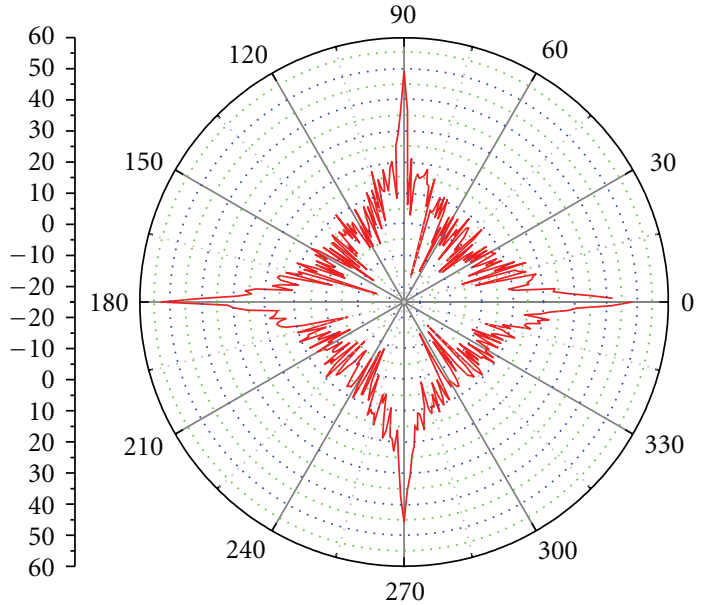

- RCS (unit: dBsm) on XOY plane

(a) $\theta=90^{\circ}, \phi=0^{\circ} \sim 360^{\circ}$

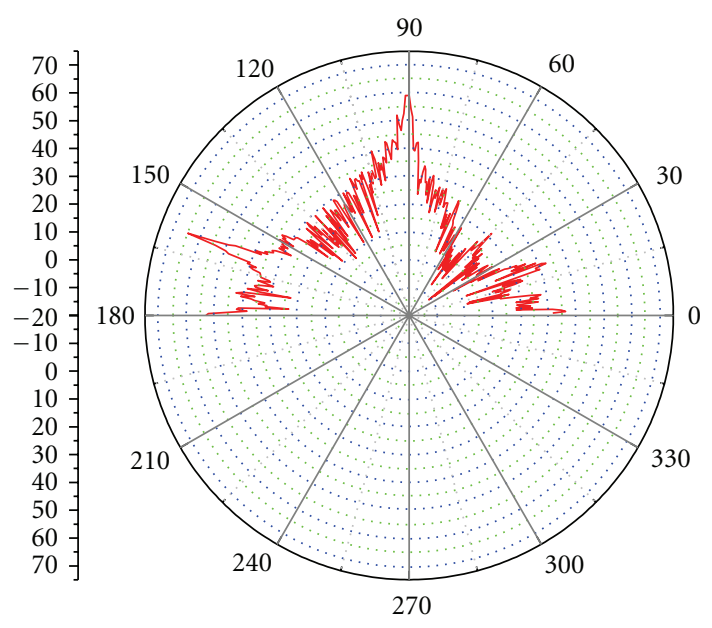

RCS (unit: dBsm), $\theta=-90^{\circ} \sim 90^{\circ}, \phi=0^{\circ}$

(b) $\theta=-90^{\circ} \sim 90^{\circ}, \phi=0^{\circ}$

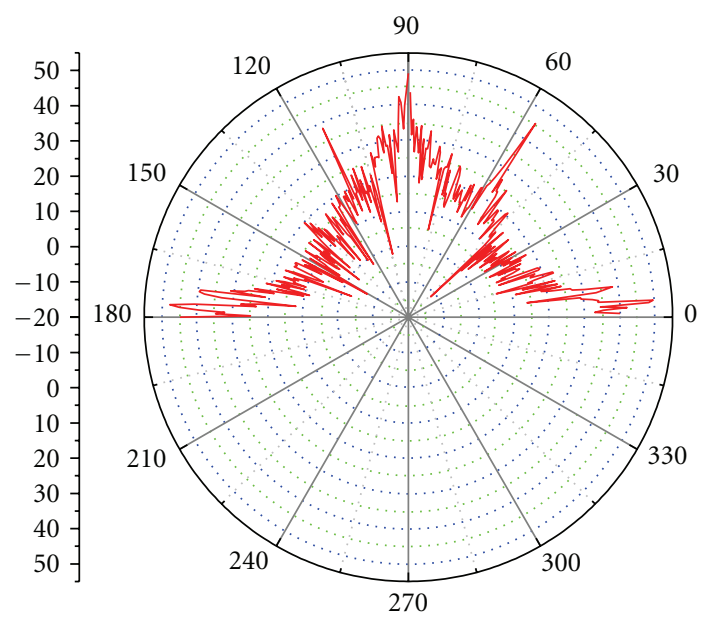

RCS (unit: dBsm), $\theta=-90^{\circ} \sim 90^{\circ}, \phi=90^{\circ}$

(c) $\theta=-90^{\circ} \sim 90^{\circ}, \phi=90^{\circ}$

FIGURe 16: 2D Monostatic RCS. 
TABLE 6: Information about the simulation.

\begin{tabular}{lcc}
\hline Project's name & Number of CPU & Wall Clock Time (Second) \\
\hline A ship on the sea & 1024 & 1366 \\
\hline
\end{tabular}

namely, it needs more than 45 hours to perform the simulation. However, only 2670 seconds is required by using 64 CPUs, which means that the computational efficiency is greatly improved. Meanwhile, the illustrating of parallel speed-up ratio in Figure 12 is linear. Namely, with more CPUs, the computation time will be reduced linearly and also the parallel speed-up ratio will be increased linearly. This is consistent with the theory that parallel PO has a linear parallel speed-up ratio [17].

Finally, the Bistatic RCS are also illustrated in Figure 13.

3.3. Large-Scale Parallel Computation by Using More Than 1000 CPUs. In this example, an electrically large model of a ship on the sea is simulated by using 1024 CPUs. The whole model consists of two parts: a ship and the sea, which are illustrated in Figure 14. The ship is a PEC model and the sea is of dielectric material whose parameters are presented in Table 5. The geometrical dimensions of the ship are $30 \mathrm{~m} \times$ $2.2 \mathrm{~m} \times 5.2 \mathrm{~m}$, and those of the sea are $59 \mathrm{~m} \times 59 \mathrm{~m}$. As the simulation frequency is $10 \mathrm{GHz}$, the ship and the sea have the electrical dimensions of $1000 \lambda \times 73.3 \lambda \times 173.3 \lambda$ and $1966.7 \lambda \times 1966.7 \lambda$, respectively. In addition, the elements of the ship generated are 740708 , and those of the sea are 8809388. Therefore, the whole model (ship + sea) has the total elements of 9550096.

Monostatic RCS is calculated. The incident wave is of $\mathrm{HH}$ $(\phi \phi)$ polarization, and the backward scattering direction is $\theta=0^{\circ} \sim 90^{\circ}, \phi=0^{\circ} \sim 360^{\circ}$ (i.e., the space above the sea).

From the description above, we can find that it is unrealistic to solve this problem by RWG MoM algorithm because of the large quantity of elements. Even if PO technique is used, the serial code will also encounter so large computational quantity that requires very long time for simulation. Thus, we employ large-scale parallel technique described in Section 2.3 and use 1024 CPUs in SSC to solve this problem. 3D and 2D results are presented in Figures 15 and 16, respectively. Finally, the computation information is listed in Table 6.

From the results presented above, we can find that the simulation time is significantly reduced by using 1024 CPUs; it only takes less than half an hour to solve this problem. In addition, it is necessary to indicate that this is the first time in China employing more than 1000 CPUs simultaneously to solve such a large problem by using large-scale parallel PO technique.

\section{Conclusion}

In this paper, a parallel method to predict RCS of electrically large NURBS model with dielectric coatings is presented. A real-life model with extra big electrical dimensions is simulated by using 1024 CPUs, which demonstrates that this paper's method is capable of solving the practical challenging problem.

\section{Acknowledgments}

This work is supported by the National Natural Science Foundation of China (61072019), the Fundamental Research Funds for the Central Universities of China (JY10000902002, K50510020017), and the Foundation of Science and Technology on Antenna and Microwave Laboratory (9140C070502110C0702). Finally, many thanks are due to Shanghai Supercomputer Center (SSC).

\section{References}

[1] W. B. Gordon, "High frequency approximation to the physical optics scattering integral," IEEE Transactions on Antennas and Propagation, vol. 42, no. 3, pp. 427-432, 1994.

[2] W. B. Gordon, "Far-field approximations to the KirchoffHelmholtz representations of scattered fields," IEEE Transactions on Antennas and Propagation, vol. 23, no. 4, pp. 590-592, 1975.

[3] N. N. Youssef, "Radar cross section of complex targets," Proceedings of the IEEE, vol. 77, no. 5, pp. 722-734, 1989.

[4] P. Pouliguen and L. Desclos, "A physical optics approach to near field RCS computations," Annals of Telecommunications, vol. 51, no. 5-6, pp. 219-226, 1996.

[5] P. H. Pathak, "High frequency techniques for antenna analysis," Proceedings of the IEEE, vol. 80, no. 1, pp. 44-65, 1992.

[6] D. J. Andersh, M. Hazlett, S. W. Lee, D. D. Reeves, D. P. Sullivan, and Y. Chu, "XPATCH: a high-frequency electromagnetic scattering prediction code and environment for complex three-dimensional objects," IEEE Antennas and Propagation Magazine, vol. 36, pp. 65-69, 1994.

[7] M. Domingo, F. Rivas, J. Perez, R. P. Torres, and M. F. Catedra, "Computation of the RCS of complex bodies modeled using NURBS surfaces," IEEE Antennas and Propagation Magazine, vol. 37, no. 6, pp. 36-47, 1995.

[8] J. Perez and M. F. Catedra, "Application of physical optics to the RCS computation of bodies modeled with NURBS surfaces," IEEE Transactions on Antennas and Propagation, vol. 42, no. 10, pp. 1404-1411, 1994.

[9] M. Domingo, F. Rivas, J. Perez, R. P. Torres, and M. F. Catedra, "Computation of the RCS of complex bodies modeled using NURBS surfaces," IEEE Antennas and Propagation Magazine, vol. 37, no. 6, pp. 36-47, 1995.

[10] J. Perez, J. Sainz, and M. F. Catedra, "Analysis of radiation and scattering of bodies modelled with parametric surfaces," in Proceedings of the AP-S International Symposium \& URSI Radio Science Meeting. Part 1 (of 3), pp. 1820-1823, July 1996.

[11] F. S. de Adana, I. González, O. Gutiérrez, and M. F. Cátedra, "Asymptotic method for analysis of RCS of arbitrary targets composed by dielectric and/or magnetic materials," IEE Proceedings: Radar, Sonar and Navigation, vol. 150, no. 5, pp. 375-378, 2003.

[12] E. E. Garrido Jr. and D.C. Jenn, "A MATLAB physical optics RCS prediction code," ACES Newsletter, vol. 15, pp. 7-12, 2000.

[13] F. Chatzigeorgiadis and D. C. Jenn, "A MATLAB physicaloptics RCS prediction code," IEEE Antennas and Propagation Magazine, vol. 46, no. 4, pp. 137-139, 2004.

[14] F. Chatzigeorgiadis, D. C. Jenn, and D. C. Schleher, Development of code for a physical optics radar cross section prediction and analysis application [M.S. thesis], Naval Postgraduate School, Monterey, Calif, USA, 2004. 
[15] Z. H. Du, Parallel Programming Technology of High Performance Computation: MPI Programming, Qinghua University Press, Beijing, China, 2001.

[16] http://www.ssc.net.cn.

[17] L. Piegl and W. Tiller, The NURBS Book, Springer, Berlin, Germany, 1997.

[18] J. Perez and M. F. Catedra, "Application of physical optics to the RCS computation of bodies modeled with NURBS surfaces," IEEE Transactions on Antennas and Propagation, vol. 42, no. 10, pp. 1404-1411, 1994.

[19] Y. Zhang, Parallel Computation in Electromagnetics, Xidian University Press, Xi'an, China, 2006.

[20] E. E. Garrido Jr, Graphical user interface for a physical optics radar cross section prediction code [M.S. thesis], Naval Postgraduate School, Monterey, Calif, USA, 2000.

[21] N. Faros, Radar cross section synthesis for planar resistive surfaces [M.S. thesis], Naval Postgraduate School, Monterey, Calif, USA, 1994.

[22] A. C. Ludwig, "Computation of radiation patterns involving numerical double integration," IEEE Antennas and Propagation Magazine, vol. 11, pp. 767-769, 1968.

[23] A. C. Ludwig, "Comments on the accuracy of the 'Ludwig," IEEE Transactions on Antennas and Propagation, vol. 36, no. 4, pp. 578-579, 1988.

[24] http://www.feko.info/.

[25] S. M. Rao, D. R. Wilton, and A. W. Glisson, "Electromagnetic scattering by surfaces of arbitrary shape," IEEE Transactions on Antennas and Propagation, vol. 30, no. 3, pp. 409-418, 1982.

[26] Y. Zhang and T. K. Sarkar, Parallel Solution of Integral Equation Based EM Problems in the Frequency Domain, John Wiley \& Sons, Hoboken, NJ, USA, 2009.

[27] http://www.EM-HOBBIES.com/.

[28] Y. Zhang, T. K. Sarkar, X. W. Zhao et al., Higher Order Basis Based Integral Equation Solver (HOBBIES), John Wiley \& Sons, Hoboken, NJ, USA, 2012. 

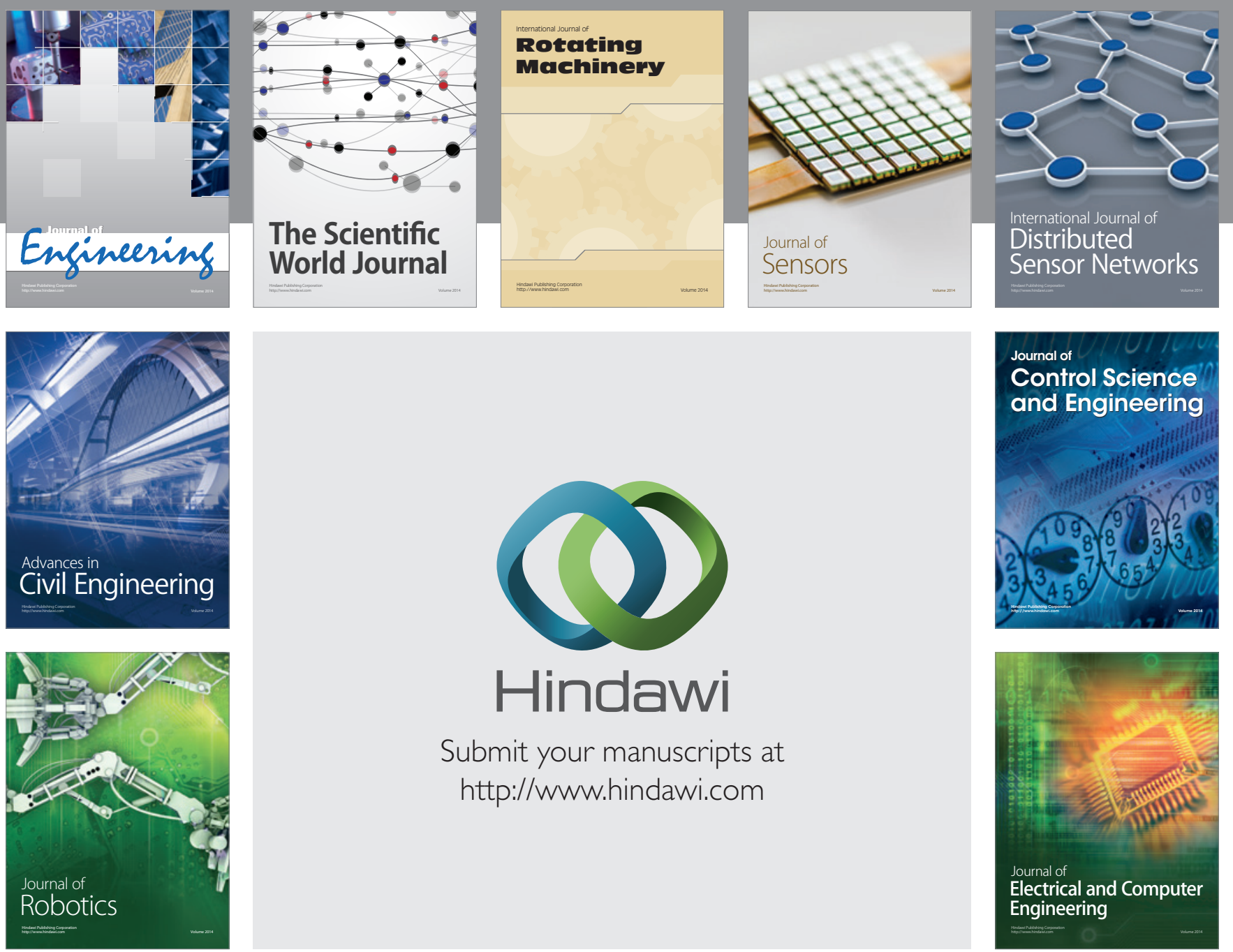

Submit your manuscripts at

http://www.hindawi.com
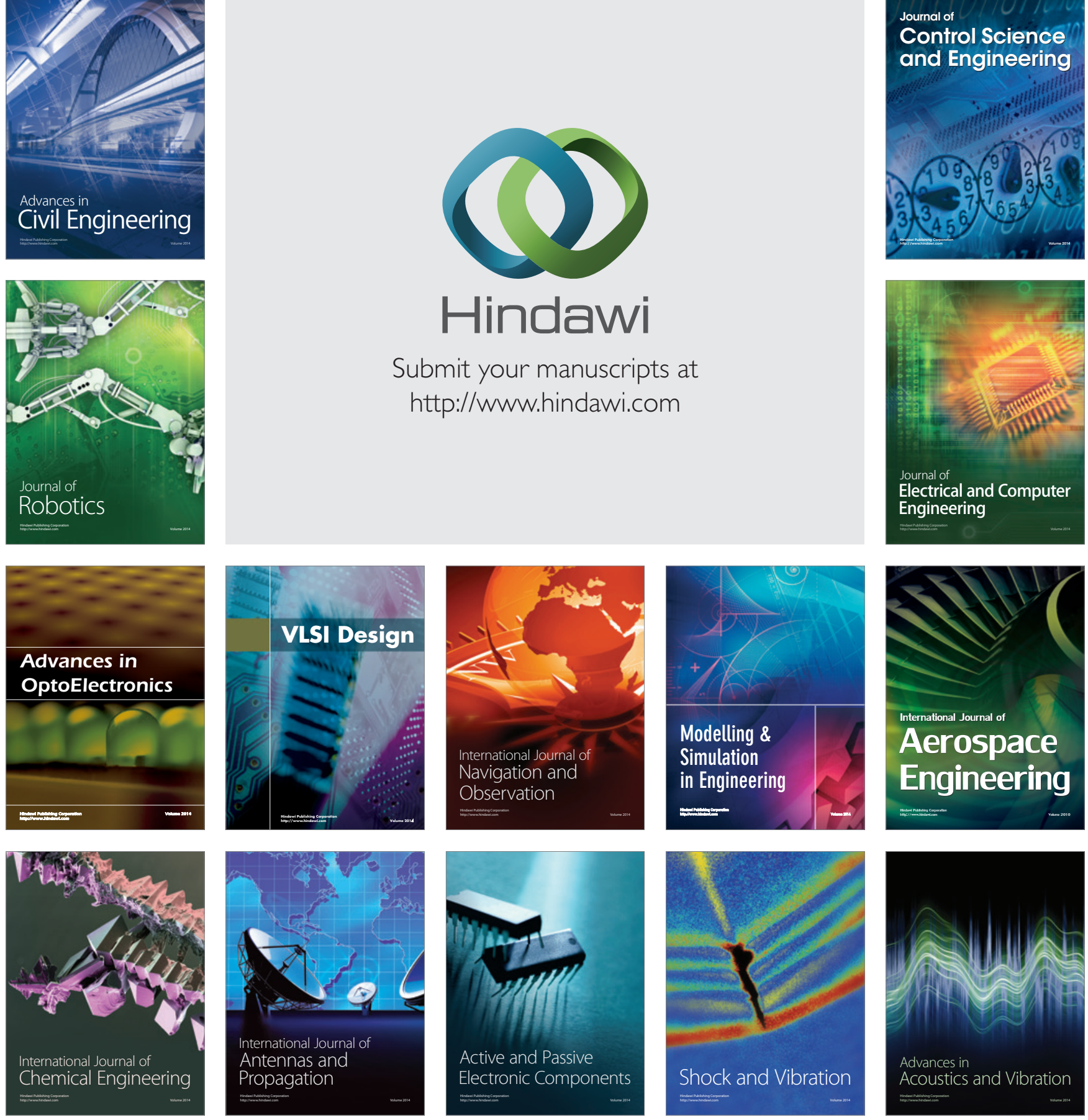